\section{Análise do impacto econômico-assistencial de duas abordagens para redução de custos em um plano odontológico de autogestão}

\author{
The impact on costs and care of two approaches \\ to reduce employees' dental plan expenses \\ in a private company
}

\footnotetext{
${ }^{1}$ Fundação de Amparo Social, Hospital Moinhos de Vento, Porto Alegre, Brasil.

2 Faculdade de Odontologia, Pontifícia Universidade Católica do Rio Grande do Sul, Porto Alegre, Brasil. 3 Faculdade de Medicina, Universidade Federal do Rio Grande do Sul, Porto Alegre, Brasil.

4 Brigada Militar do Rio Grande do Sul, Porto Alegre, Brasil.

Correspondência L. C. Costa Filho

Fundação de Amparo Social, Hospital Moinhos de Vento. Av. Loureiro da Silva 2001, sala 409, Porto Alegre, RS 90050-240, Brasil. periodontia@rocketmail.com
}

\begin{abstract}
The present study evaluated the dental care plan offered to 4,000 employees of a private hospital and their respective families. The analysis covered three stages: (1) baseline (control), when dental care was provided by an outsourced company with a network of dentists paid for services, (2) a renegotiation of costs with the original dental care provider, and (3) provision of dental care by the hospital itself, through directly hired dentists on regular salaries. Monthly economic and clinical data were collected for this research. The dental plan renegotiation reduced costs by $37 \%$ in relation to baseline, and the hospital's own dental service reduced costs by 50\%. Renegotiation led to a $31 \%$ reduction in clinical procedures, without altering the dental care profile; the hospital's own dental service did not reduce the total number of clinical procedures, but modified the profile of dental care, since procedures related to the causes of diseases increased and surgical/restorative procedures decreased.
\end{abstract}

Health Planning, Cost Saving; Deutar Insurence; Oral Health

\author{
Luiz Cesar da Costa Filho 1,2 \\ Bruce Bartholow Duncan ${ }^{3}$ \\ Carisi Anne Polanczyk 3 \\ Marina Lara Sória 1 \\ Ana Paula Habekost 2 \\ Carolina Covolo da Costa 4
}

\section{Introdução}

Os gastos com planos de saúde têm se tornado um desafio gerencial para as empresas, pois seus custos sobem acima da inflação, basicamente por dois motivos: a população está ficando mais velha, e isso demanda mais serviços de saúde; e a disponibilidade tecnológica na área da saúde está aumentando, o que gera novos serviços e a possibilidade de tratamentos mais precoces 1,2 Ao mesmo tempo, o plano de saúde é um dos benefícios mais valorizados pelos funcionários. Atualmente as empresas buscam formas de reduzir os custos com a saúde e manter, ou até mesmo ampliar, os benefícios oferecidos para que a satisfação dos funcionários não seja afetada 3,4 .

Dentro da oferta de saúde pelas empresas, o setor que mais vem crescendo no Brasil é o da odontologia 5,6. Além disso, segundo a Lei $n^{\circ}$. 3.520/04, já aprovada pela Comissão de Seguridade Social e Família do Congresso Nacional, empresas com mais de 500 funcionários poderão ser obrigadas a manter um setor de odontologia do trabalho ${ }^{7}$.

É sabido que as formas de prestação de serviço bucal (taxas por serviço, odontologia de grupo, capitação, serviço próprio, reembolso, salário etc.) têm impacto direto nos custos e no perfil assistencial oferecido aos pacientes 8,9,10,11. Em virtude desse fato, o setor da saúde e as empresas devem saber quais modalidades geram mais benefícios aos pacientes com menor custo, e com 
isso o uso dos recursos poderá ser otimizado sem diminuir a satisfação dos usuários.

Neste estudo avaliaram-se os impactos no perfil assistencial, no custo, na cobertura e na satisfação do usuário, de duas estratégias de redução de despesas dos serviços de assistência em odontologia, cobrindo cerca de 4 mil funcionários e dependentes do Hospital Moinhos de Vento pela sua Fundação de Amparo Social (FAS-HMV). Para tal avaliação, partimos da situação original do plano, quando o mesmo era terceirizado e a prestadora de serviço odontológico operava com uma rede credenciada remunerada com taxas por serviço. Contrastamos os resultados das duas abordagens distintas: a primeira, na qual aconteceu apenas uma renegociação dos preços com a prestadora original e a segunda, na qual a FAS-HMV montou um serviço de odontologia próprio que permitiu estratégias de diminuição de procedimentos sem base científica clara e favorecimento de abordagens preventivas.

\section{Métodos}

\section{O serviço odontológico da FAS-HMV}

A FAS-HMV foi criada em 1995 com o objetivo de implantar um plano de saúde de autogestão para os colaboradores do HMV e entidades a ele vinculadas. O plano também beneficia os cônjuges e dependentes até 21 anos de idade, funcionários aposentados e ex-funcionários na forma da $L e i$ $n^{o}$. 9.656/98 12 .

Em 1995, quando o plano de saúde da FASHMV foi instituído, a odontologia era terceirizada por uma empresa prestadora de serviço odontológico que operava com uma rede de profissionais credenciados que eram remunerados através do sistema de remuneração por serviço. Em meados de 2002, outra empresa de odontologia foi contratada para fazer um levantamento epidemiológico da população de usuários da FAS-HMV e com isso avaliar a possibilidade de implantação de um serviço de odontologia próprio.

Em fevereiro de 2003, junto com outras mudanças na estrutura do plano FAS-HMV, o serviço próprio de odontologia foi implantado. A proposta do serviço de odontologia próprio foi de enfocar a promoção de saúde bucal, ou seja, além de suprir as necessidades clínicas do paciente, enfatizar a prevenção e a educação em saúde 13 . Outra característica desse serviço é que os protocolos clínicos foram elaborados com uma metodologia de práticas em saúde embasadas em evidência para otimizar o benefício decorrente dos recursos financeiros do plano 14,15,16,17,18. O serviço próprio funciona com três consultórios odontológicos, sete dentistas (sendo um deles o coordenador da odontologia desse serviço), uma técnica em higiene dental e duas auxiliares de consultório dentário. O perfil dos dentistas selecionados para esse serviço foi de profissionais com boa formação como clínicos gerais, mas que possuíssem diferentes especialidades (dentística, odontopediatria, ortodontia, endodontia, periodontia e cirurgia) para que a resolutividade de casos complexos dentro do próprio serviço fosse aumentada. A equipe de profissionais escolhida também tem como característica o conhecimento dos conceitos de odontologia embasada em evidência para que também possam contribuir na elaboração dos protocolos clínicos. A técnica em higiene dental e as auxiliares de consultório dentário do serviço têm uma formação voltada para execução de procedimentos de adequação inicial em saúde bucal (raspagem supragengival e educação em saúde bucal).

Diferente do sistema anterior, a forma de remuneração dos profissionais do serviço é com base em valores fixos (contratados pela CLT - Consolidação das Leis do Trabalho - ou como pessoa jurídica).

\section{Delineamento da pesquisa}

O estudo é uma coorte retrospectiva e utiliza dados secundários coletados na FAS-HMV de janeiro de 2000 a junho de 2005.

\section{População e amostra}

Esse estudo envolve a população de usuários do plano de saúde FAS-HMV. O plano de saúde dessa fundação tem cerca de 4 mil usuários (o número é levemente variável de acordo com entradas e saídas de pessoas do plano). Os usuários são funcionários (ativos, aposentados ou ex-funcionários) do HMV e entidades vinculadas ao hospital, bem como seus cônjuges e dependentes até 21 anos de idade.

Três grupos de observações foram montados. Esses grupos se referem à linha de base e aos dois momentos de modificação no plano de atendimento da FAS-HMV: (1) linha de base-de janeiro de 2000 a fevereiro de 2001 (14 meses de observação), quando os serviços de odontologia eram terceirizados por uma empresa, a qual prestava assistência odontológica através do sistema de rede credenciada e remunerava seus profissionais através de taxas por serviço; (2) serviço com renegociação de preços - de março de 2001 a dezembro de 2002 (22 meses de observação), quando os serviços de odontologia eram prestados pela mesma empresa da linha de base, porém 
houve uma renegociação de preços que forçou a empresa a tomar medidas restritivas de acesso à rede credenciada; (3) serviço próprio - de março de 2003 a junho de 2005 (28 meses de observação), quando se dispensou a prestadora terceirizada, e os serviços de odontologia passaram a ser prestados por um serviço próprio de odontologia fornecido diretamente pela FAS-HMV.

\section{Coleta dos dados}

Os dados sobre os procedimentos realizados nos anos de 2000, 2001 e 2002 foram fornecidos pela empresa que fazia a assistência odontológica da FAS-HMV com rede credenciada antes do serviço próprio, bem como as pesquisas de satisfação dos usuários nos anos de 2000 e 2001.

Os dados sobre os procedimentos realizados no período de março de 2003 a junho de 2005 foram levantados nos prontuários clínicos do atual serviço próprio de odontologia da FAS-HMV. As pesquisas de satisfação do serviço nesse período foram obtidas pela fundação através de questionários.

Os dados sobre os custos da odontologia em todo período de janeiro de 2000 a junho de 2005 foram obtidos na empresa responsável pela gestão do plano de saúde da FAS-HMV desde sua criação em 1995. Na composição dos custos antes do serviço próprio foram considerados os custos diretos: (1) o valor pago à operadora de saúde; (2) os gastos com urgências odontológicas; (3) os gastos com consultas especializadas fora da rede da operadora; (4) os gastos com bloco cirúrgico, internações hospitalares e exames e os custos indiretos; (5) relativos a salários e encargos com dias de afastamento gerados por motivos odontológicos. E na composição dos custos do serviço próprio foram considerados: (1) o gasto com salários e encargos da equipe odontológica; (2) o gasto com a aquisição de materiais e manutenção de equipamentos; (3) os gastos com urgências odontológicas; (4) os gastos com consultas especializadas fora do serviço próprio; (5) os gastos com bloco cirúrgico, internações hospitalares e exames; (6) os custos fixos do serviço (água, luz, telefone, aluguel, limpeza etc.); (7) custo com depreciação de equipamentos e da obra civil; (8) custo com o passivo trabalhista (calculou-se o pior cenário possível com base nas informações da taxa de renovação dos funcionários do hospital, dos pagamentos de leis sociais e dos pagamentos de reclamatórias na justiça) e os custos indiretos; (9) relativos a salários e encargos com dias de afastamento gerados por motivos odontológicos.

\section{Análise estatística e forma de apresentação dos dados}

Vale ressaltar que o ponto de vista da análise econômica é o da instituição patrocinadora (FAS-HMV).

As informações sobre procedimentos feitos e custos foram processadas nos programas Microsoft Excel 2003 (Microsoft Corp., Estados Unidos) e SPSS 10.0.1 (SPSS Inc., Chicago, Estados Unidos).

Para melhorar a comparabilidade devido a variações no número de usuários, os resultados foram ajustados para uma população de $4 \mathrm{mil}$ usuários.

Os dados sobre custos foram ajustados mensalmente pelo Índice Nacional de Preços ao Consumidor (IPCA: amplo - preços monitorados; plano de saúde) e foram apresentados em reais com correção para julho de 2005. As séries históricas sobre esse indicador foram obtidas através da página eletrônica do Banco Central do Brasil (Sistema Gerenciador de Séries Temporais; http://www4.bcb.gov.br/pec/series/port, acessado em 07/Mar/2007).

As comparações entre as duas estratégias de organização do serviço foram feitas através da análise de variância e através do teste de Dunnett contra um controle (a linha de base sendo o controle) e o teste t de Dunnett foi aplicado para o caso de variâncias diferentes. As diferenças foram consideradas significativas ao nível de 5\%.

\section{Aspectos éticos}

Essa pesquisa fez uso de forma sigilosa de dados contidos em prontuários e seguiu rigorosamente as Resoluções $n^{o}$. 196/96 e $n^{\circ}$. 251/97 para pesquisas epidemiológicas do Conselho Nacional de Saúde. Essa pesquisa foi aprovada pelo Comitê de Ética em Pesquisa e Comissão Científica do Instituto de Educação e Pesquisa do Hospital Moinhos de Vento (CEP IEP-HMV) sob o número de registro 2005/38.

\section{Resultados}

A Tabela 1 mostra o número de procedimentos nos quatro grandes grupos de procedimentos: (1) diagnóstico (consultas de exame clínico, exames de raios-X, biópsias); (2) prevenção (sessões de orientação de higiene bucal, aplicações de flúor, profilaxias e aplicações de selantes); (3) adequação do meio bucal (sessões de raspagem supra e subgengival; reparo de restaurações: remoção de excessos, repolimento e pequenos consertos; tratamentos expectantes: restaurações provi- 
Médias mensais e desvios-padrão (DP), e percentual mensal médio de procedimentos; diagnóstico, preventivos, de adequação do meio bucal, restauradores e outros.

\begin{tabular}{|c|c|c|c|c|c|c|}
\hline \multirow[t]{2}{*}{ Procedimentos } & \multicolumn{2}{|c|}{ Linha de base } & \multicolumn{2}{|c|}{ Renegociação } & \multicolumn{2}{|c|}{ Serviço próprio } \\
\hline & Média mensal $\pm \mathrm{DP}$ & $\%$ & Média mensal $\pm \mathrm{DP}$ & $\%$ & Média mensal $\pm \mathrm{DP}$ & $\%$ \\
\hline Diagnóstico & $482 \pm 51$ & 34,05 & $372 \pm 127$ * & 38,22 & $409 \pm 42$ * & 27,35 \\
\hline Prevenção & $352 \pm 41$ & 24,90 & $220 \pm 98$ * & 22,58 & $520 \pm 104$ * & 34,81 \\
\hline Adequação & $98 \pm 27$ & 6,94 & $48 \pm 17$ * & 4,97 & $314 \pm 74$ * & 21,04 \\
\hline Restauradores & $378 \pm 58$ & 26,71 & $247 \pm 85$ * & 25,35 & $109 \pm 25$ * & 7,26 \\
\hline Outros & $104 \pm 19$ & 7,40 & $87 \pm 27$ & 8,88 & $142 \pm 31$ * & 9,54 \\
\hline Total & $1.414 \pm 160$ & 100,0 & $974 \pm 325$ * & 100,0 & $1.494 \pm 207$ & 100,0 \\
\hline
\end{tabular}

* Diferente da linha de base: $p<0,01$.

sórias com ionômero de vidro ou restaurador intermediário - IRM e; tratamento restaurador atraumático) e (4) restaurador (colagem de fragmentos e restaurações de resina e amálgama) - na linha de base e durante o período das duas estratégias de redução de custos. Houve diferenças significativas dos dois tipos de abordagens para redução de custo em relação ao controle (linha de base). A renegociação de preços causou a redução nos quatro grupos de procedimentos em relação ao controle. Já a implantação do serviço próprio teve uma leve redução nos procedimentos diagnósticos, houve aumento marcante nos procedimentos preventivos e nos procedimentos de adequação do meio bucal, e houve uma redução acentuada nos procedimentos restauradores.

Ainda na Tabela 1, quando se observa o percentual dos principais procedimentos, nota-se que a distribuição percentual dos serviços é muito semelhante na linha de base e no momento da renegociação, ou seja, apesar da redução numérica no total de serviços observada, o tipo de assistência é muito semelhante e contempla menos os procedimentos de adequação do meio bucal, ou seja, raspagem supra e subgengival, restaurações provisórias, tratamentos restauradores atraumáticos e recuperação de restaurações. Já no serviço próprio, a distribuição percentual de cada tipo de serviço é bem diferente dos momentos anteriores, e os serviços menos contemplados são os restauradores.

A Tabela 2 trata de serviços específicos de baixa prevalência nesse plano de saúde (menos de $8 \%$ do total de serviços realizados). Na maioria dos serviços não houve diferença entre a linha de base e a renegociação de preço, a exceção se fez no número de exodontias de dentes decíduos e no número de cirurgias de pequeno porte que di- minuíram após a renegociação. Já a implantação do serviço próprio trouxe mudanças significativas em relação à linha de base.

A Tabela 3 mostra que o número de procedimentos diminuiu quando da renegociação de preços, indicando que esta estratégia de redução de custo fez com que o prestador adotasse medidas restritivas no atendimento, mas o perfil dos procedimentos realizados, como demonstrado na Tabela 1, continuou o mesmo. Já a implantação de um serviço próprio não reduz o número total de procedimentos em relação ao controle (Tabela 3), mas modifica o perfil assistencial.

Quanto à redução de custo, tanto a renegociação quanto o serviço próprio foram capazes de reduzir custos, mas o serviço próprio conseguiu uma maior economia (Tabela 3). Porém se considerarmos o custo por procedimento, o serviço próprio conseguiu uma redução de mais de $50 \%$ no preço do procedimento enquanto a renegociação não teve efeito no preço por procedimento. Deve-se lembrar aqui que o custo por procedimento é do ponto de vista da instituição patrocinadora (FAS-HMV) e não o custo real por procedimento sob a ótica da operadora terceirizada.

Os investimentos com instalações e equipamentos para o início do serviço próprio (os quais totalizaram 157.215 Reais - com valores ajustados pelo o IPCA - planos de saúde - corrigidos para julho de 2005) foram computados mensalmente em forma de depreciação de móveis, de equipamentos e da obra civil. Também foi computada nos custos uma constante mensal sobre os riscos de passivo trabalhista imaginando-se o pior cenário possível, o que deu em valores ajustados 3.060 Reais. Porém, o valor real desses primeiros 28 meses de funcionamento foi de 154 Reais por mês (cerca de $5 \%$ do pior cenário imaginado). A 
Médias mensais e desvios-padrão (DP) de outros procedimentos ofertados pelo plano odontológico.

\begin{tabular}{|c|c|c|c|}
\hline Procedimentos & $\begin{array}{c}\text { Linha de base } \\
\text { Média mensal } \pm \mathrm{DP}\end{array}$ & $\begin{array}{c}\text { Renegociação } \\
\text { Média mensal } \pm \text { DP }\end{array}$ & $\begin{array}{c}\text { Serviço próprio } \\
\text { Média mensal } \pm \text { DP }\end{array}$ \\
\hline Endodônticos & $26 \pm 10$ & $21 \pm 9$ & $17 \pm 8$ * \\
\hline Exodontias & $39 \pm 10$ & $34 \pm 15$ & $24 \pm 11$ * \\
\hline Endodontias pediátricas & $1,2 \pm 0,3$ & $0,6 \pm 0,1$ & $1,2 \pm 0,2$ \\
\hline Exodontias pediátricas & $13 \pm 9$ & $5 \pm 6$ * & $12 \pm 5$ \\
\hline Tratamentos oclusais & $0,1 \pm 0,3$ & $0,1 \pm 0,5$ & $47 \pm 11$ * \\
\hline Cirurgias especializadas & $4 \pm 3$ & $2 \pm 2$ & $7 \pm 4$ \\
\hline Tratamentos fora do sistema & $5 \pm 3$ & $5 \pm 3$ & $1 \pm 2$ * \\
\hline Tratamentos de urgência & $17 \pm 6$ & $19 \pm 8$ & $34 \pm 9$ * \\
\hline
\end{tabular}

* Diferente da linha de base: $p<0,01$.

Tabela 3

Médias mensais e desvios-padrão (DP) dos custos (em Reais, julho de 2005) e do número total de procedimentos.

\begin{tabular}{|c|c|c|c|}
\hline \multirow[t]{2}{*}{ Procedimentos } & Linha de base & Renegociação & Serviço próprio \\
\hline & Média mensal $\pm \mathrm{DP}$ & Média mensal $\pm \mathrm{DP}$ & Média mensal $\pm \mathrm{DP}$ \\
\hline \multicolumn{4}{|l|}{ Custos } \\
\hline Gasto com a operadora de saúde & $58.308 \pm 1.433$ & $34.284 \pm 847$ * & n.a. \\
\hline Gasto com urgências & n.a. & $502 \pm 330$ & $271 \pm 408$ \\
\hline Pagamento da equipe odontológica & n.a. & n.a. & $16349 \pm 1.655$ \\
\hline Material e equipamentos & n.a. & n.a. & $5.496 \pm 2.996$ \\
\hline Consultas especializadas fora do sistema & $179 \pm 93$ & $161 \pm 119$ & $34 \pm 62$ * \\
\hline Gastos hospitalares & $1.982 \pm 2.680$ & $3.075 \pm 4.992$ & $788 \pm 1.605$ \\
\hline Custo da depreciação & n.a. & n.a. & $1.503 * \star$ \\
\hline Custos fixos & n.a. & n.a. & 1.550 ** \\
\hline Custo indireto dos afastamentos & $608 * \star$ & 608 ** & $1.161 \star \star$ \\
\hline Passivo trabalhista & n.a. & n.a. & $3.060 * \star$ \\
\hline Custo total & $61.077 \pm 3.235$ & $38.630 \pm 5.186 *$ & $30.212 \pm 3.853$ * \\
\hline \multicolumn{4}{|l|}{ Procedimentos } \\
\hline Total de procedimentos & $1.414 \pm 160$ & $974 \pm 325$ * & $1.494 \pm 207$ \\
\hline Custo por procedimento & $43,6 \pm 4,9$ & $44,5 \pm 17,4$ & $20,5 \pm 3,5$ * \\
\hline
\end{tabular}

n.a.: custo não aplicável.

* Diferente da linha de base: $p<0,01$;

** Constantes estimadas com base em informações do hospital ou com informações teóricas.

escolha do pior cenário se deu pelo fato de que o passivo trabalhista aumenta com o passar dos anos.

A Tabela 4 mostra duas avaliações de satisfação realizadas com o mesmo método de amostragem aleatória. Uma das avaliações foi realizada na época da renegociação e outra na época do serviço próprio, e pode-se observar que o serviço próprio foi mais bem avaliado.
As Figuras 1, 2 e 3 mostram a tendência temporal dos custos, do número de procedimentos e dos custos por procedimento. As linhas de tendência de cada grupo foram realizadas pela função Lowess com três interações e com $50 \%$ dos pontos utilizados para o ajuste.

A Figura 1 mostra os custos da odontologia de uma forma temporal. Nota-se claramente a redução dos custos em relação à situação origi- 
Duas pesquisas de opinião sobre o plano odontológico realizadas através amostragem aleatória. Uma pesquisa foi realizada em julho de 2002 (período da renegociação) e outra foi realizada em julho de 2005 (período do serviço próprio)

\begin{tabular}{|c|c|c|c|c|}
\hline & \multirow{2}{*}{\multicolumn{3}{|c|}{$\begin{array}{l}\text { Pesquisa de opinião sobre o plano odontológico } \\
\text { Julho de } 2002\end{array}$}} & \multirow[b]{2}{*}{2005} \\
\hline & & & & \\
\hline & $\%$ & IC95\% & $\%$ & $1 \mathrm{C} 95 \%$ \\
\hline Elogio & 7,0 & $2,6-14,6$ & 37,0 & $23,2-52,5$ * \\
\hline Reclamação & 27,9 & $18,8-38,6$ & 6,5 & $1,4-17,9$ * \\
\hline Sem comentário & 65,1 & $54,1-75,1$ & 56,5 & $41,1-71,1$ \\
\hline
\end{tabular}

* Diferença estatística entre as duas pesquisas: $p<0,01$.

Figura 1

Evolução temporal dos custos mensais do plano odontológico (os dados são apresentados padronizados para um serviço atendendo 4 mil usuários e ajustado pelo Índice Nacional de Preços ao Consumidor - IPCA).

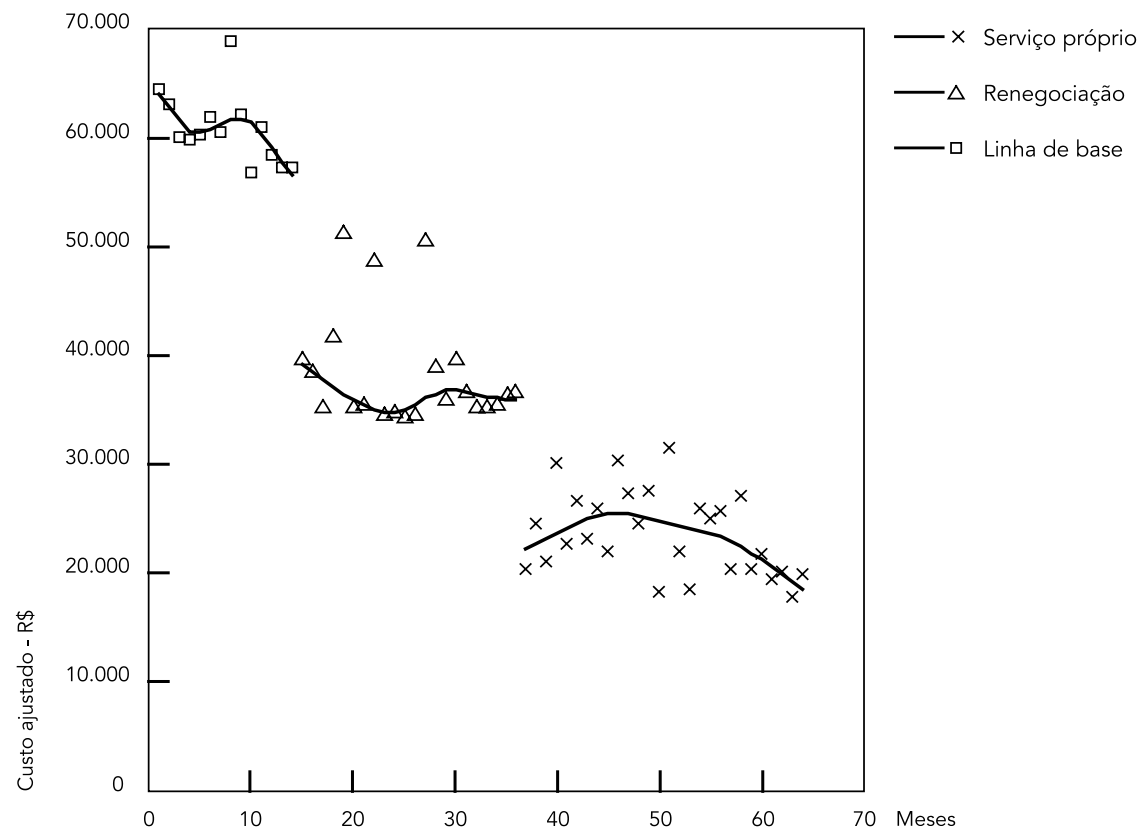

nal (linha de base) na renegociação e no serviço próprio. Existem picos eventuais de alta nos custos, os quais estão correlacionados a cirurgias e a internações em ambiente hospitalar.

Na Figura 2, nota-se no momento da renegociação (a partir de março de 2001 - mês 15) que houve uma queda brusca no número de proce- dimentos realizados, mas a partir de fevereiro de 2002 (mês 26) começou uma tendência de aumento no número de procedimentos que chegou a níveis semelhantes ao da linha de base (a partir de junho de 2002 - mês 30). Já o serviço próprio manteve um grande número de atendimentos. 
Figura 2

Evolução temporal do número total de procedimentos realizados no plano odontológico (os dados são apresentados padronizados para um serviço atendendo 4 mil usuários).

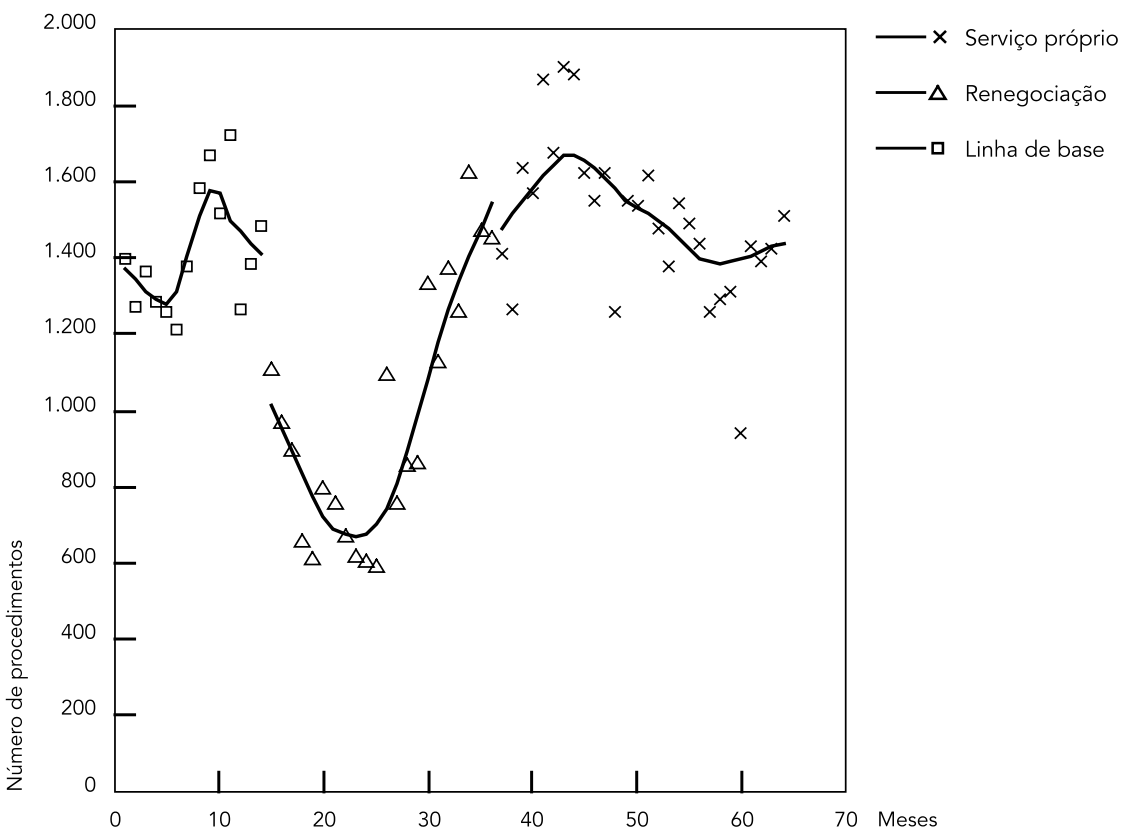

Figura 3

Evolução temporal do custo por procedimento no plano odontológico (os dados são apresentados padronizados para um serviço atendendo 4 mil usuários e ajustado pelo Índice Nacional de Preços ao Consumidor - IPCA).

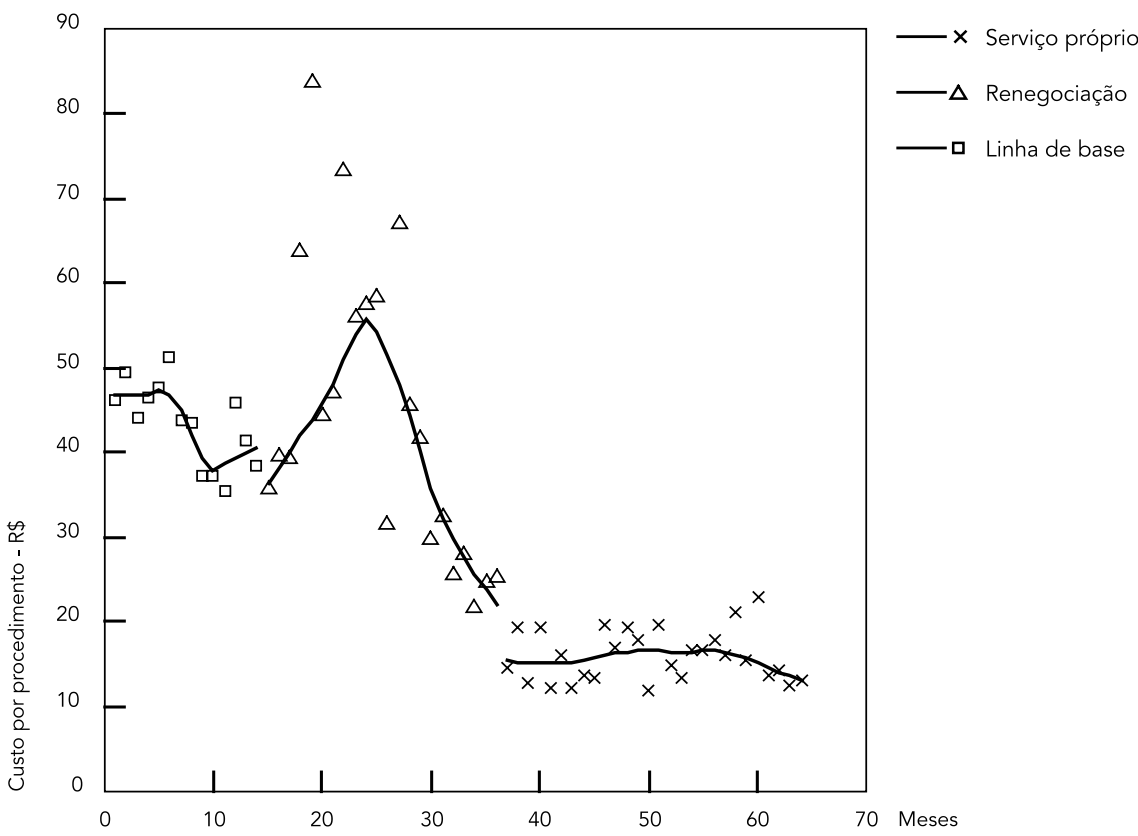


A Figura 3 mostra a tendência temporal no custo por procedimento. Durante o período da renegociação há um aumento no preço do procedimento para o patrocinador, seguido de uma queda acentuada, e isso ocorreu pelo fenômeno demonstrado na Figura 2, ou seja, a variabilidade no número de procedimentos durante a vigência da estratégia de renegociação causou a variabilidade no preço por procedimento, uma vez que a quantidade paga à operadora terceirizada era fixa.

\section{Discussão}

O presente estudo avaliou, dentro de um mesmo plano de saúde odontológico, o impacto causado na assistência por duas formas de redução de custo, a renegociação de preços com uma prestadora que operava com rede credenciada e a implantação de um serviço odontológico próprio com orientação de odontologia baseada em evidências e promoção de saúde.

As duas estratégias adotadas conseguiram diminuir os custos da odontologia, enquanto a situação original consumia $14,6 \%$ dos recursos totais do plano de saúde, com a renegociação esse percentual baixou para 10,4\% e com o serviço próprio chegou a 7,7\% do total de recursos. Quanto aos procedimentos, a renegociação basicamente diminuiu o número de procedimentos sem alterar o perfil da assistência, enquanto que o serviço próprio não reduziu a quantidade de serviços prestados, mas modificou o perfil assistencial.

Vários autores abordam de forma teórica a questão de formas de remuneração na odontologia $8,11,19,20$, mas poucas vezes se relataram testes de um sistema de remuneração em detrimento de outro 9,21,22,23. Ao sistema de taxas por serviço é atribuído o fato de aumentar a indução de demanda e com isso onerar o sistema, e ao mesmo tempo essa forma de atuação não estimularia ações de promoção de saúde 10 . Já ao sistema de capitação, no qual o dentista recebe por adesão, é imputado o fato de que o mesmo geraria mais ações preventivas e menos ações restauradoras ${ }^{11}$. E ao sistema de remuneração por salários é imputado o fato de queda na produtividade ${ }^{8,9}$. No presente estudo, o sistema de remuneração por salários do serviço próprio não implicou queda de produtividade em relação ao sistema original; possivelmente porque os estudos que avaliaram o sistema de remuneração por salários o tenham estudado no setor público, onde há maior estabilidade no emprego 9,19. Já a renegociação de preços gerou uma queda no número de procedimentos em um primeiro momento, indicando que a prestadora de serviços odontológicos adotou medidas restritivas que pudessem manter sua margem de lucro.

Existe uma clara mudança de paradigma de tratamento com a implantação do serviço próprio, o qual valoriza tratamentos relacionados a causas das doenças (prevenção e adequação do meio bucal) e restringe o tratamento restaurador. Essa diferença talvez se explique pelo fato desse serviço próprio ter seus protocolos clínicos embasados em evidência, e com forte ênfase na odontologia de promoção de saúde 13,24; ou também porque alguns procedimentos de adequação, como tratamentos restauradores atraumáticos 25 e reparo de restaurações (remoção de excessos, repolimentos e resselamentos) substituíram a troca de restaurações. A pequena diminuição dos serviços de diagnóstico observada no serviço próprio se deu pela diminuição do número de consultas iniciais, provavelmente porque os pacientes em um serviço de rede credenciada (linha de base) eram examinados por diferentes profissionais cada vez que trocavam de especialidade, coisa que não ocorre em um sistema com serviço próprio, onde todos os profissionais têm acesso ao mesmo prontuário.

Por vezes os serviços com grupos fechados, como o sistema de capitação, são acusados de negligenciar os tratamentos 21; mas provavelmente não é isso que ocorre com os procedimentos restauradores do serviço próprio, pois, no sistema de capitação, quem assume o risco financeiro é o dentista e, no caso desse serviço próprio, quem assumiu o risco financeiro foi a empresa, a qual não influenciou na tomada de decisão dos dentistas. Outra evidência de que não houve negligência no serviço próprio é observada no fato de que o número total de procedimentos não foi diminuído em comparação com a situação original.

A renegociação não provocou mudanças significativas para os serviços de baixa prevalência. No serviço próprio houve os seguintes acontecimentos:

- Uma diminuição no número de endodontias, provavelmente porque o aumento de procedimentos de adequação como tratamentos restauradores atraumáticos e a diminuição do número de restaurações tenham diminuído as invasões ao tecido pulpar;

- Uma diminuição no número de exodontias, provavelmente porque aumentaram as cirurgias periodontais especializadas com finalidade de restabelecer as distâncias biológicas (isso gerou um maior aproveitamento de raízes), e pela adoção de protocolos mais conservadores de exodontia de dentes retidos 26 ;

- Um aumento grande nos tratamentos oclusais devido à introdução de duas novas áreas de atu- 
ação não cobertas anteriormente: a ortodontia preventiva e o tratamento das disfunções têmporo-mandibulares. Na linha de base o único tratamento oclusal oferecido era o ajuste oclusal;

- Uma diminuição da utilização do bloco cirúrgico hospitalar no serviço próprio, principalmente pela conduta mais conservadora na exodontia de dentes retidos 26 e por uma reavaliação mais criteriosa na indicação de cirurgias ortognáticas para tratamento de disfunções têmporo-mandibulares;

- Uma diminuição na utilização de serviços especializados fora do sistema do plano de saúde, provavelmente porque o serviço próprio foi montado com base em levantamento epidemiológico prévio e isso possibilitou a contratação de dentistas com habilidades capazes de resolver a demanda de serviços mais especializados;

- Um aumento nos atendimentos de urgência, provavelmente porque as urgências foram absorvidas pelos dentistas do serviço próprio e a população de usuários tem um vínculo forte com esses dentistas, coisa que não acontecia com o serviço de urgência terceirizado que vigorava na linha de base e durante o período renegociado. Outra possível explicação é a facilidade de acesso dos funcionários ao serviço próprio, pois esse serviço está alocado dentro do próprio hospital. Ou talvez até mesmo as diferenças estejam na codificação das urgências (na linha de base ou durante o período renegociado os dentistas poderiam ter codificado atendimentos de urgência como endodontia ou restaurações).

O serviço próprio se tornou mais resolutivo que a situação original, pois aumentou a realização de serviços mais especializados e diminuiu a utilização de serviços fora do sistema e de bloco cirúrgico hospitalar. A maior resolutividade do serviço próprio talvez venha do fato de o serviço ter sido montado com base no conhecimento prévio das necessidades daquela população de usuários. Os procedimentos hospitalares, embora pouco freqüentes (Tabela 2), têm um grande impacto nos custos (Tabela 3).

A principal reclamação do serviço próprio foi o tempo de espera para o atendimento, pois um serviço próprio diminui a disponibilidade de profissionais; e o principal elogio foi a qualificação e o acolhimento da equipe odontológica. No momento da renegociação as principais queixas foram em relação aos atendimentos de odontopediatria.

A diminuição do preço pago pela FAS-HMV à prestadora de serviços odontológicos após a renegociação dos preços e o crescimento do número de atendimentos no final do período da renegociação culminou na implantação do serviço próprio em março de 2003, pois a relação comer- cial ficou muito desfavorável para a prestadora de serviço odontológico. A redução adicional nos custos conseguida pelo serviço próprio se deu pelos seguintes motivos: remoção do componente de lucro da empresa anterior; diminuição na indução de demanda, pois os dentistas não são remunerados pela quantidade de serviço; pela mudança no perfil assistencial; e pela otimização de recursos através da implantação de condutas clínicas embasadas em evidência 18,27.

É importante ressaltar as limitações metodológicas desse estudo:

- Os dados clínicos da linha de base e do período renegociado foram fornecidos pela empresa que fazia assistência odontológica com sistema de rede credenciada e os dados do serviço próprio foram coletados diretamente nos prontuários por um profissional não relacionado ao serviço. Essa diferença de métodos de coleta pode ter gerado algumas distorções na captação dos dados;

- Este estudo não é um ensaio clínico aleatório e sim uma análise retrospectiva de um plano de saúde de uma empresa. Embora a população seja na sua maior parte a mesma, existe uma taxa de renovação no plano que pode influenciar os resultados;

- Não existem estudos controlados similares comparando os efeitos dessas duas abordagens de redução de custo em planos odontológicos, o que faz desse um caso isolado que inicia uma nova discussão. Logo, mais estudos são necessários para determinar os fatores que influenciam essas abordagens em diferentes realidades e diferentes populações;

- As observações são de natureza ecológica, logo se deve tomar cuidados com inferências no nível individual.

Apesar das limitações metodológicas, o presente estudo nos permite observar que a renegociação para diminuição de preços em um sistema de atenção à saúde bucal terceirizado com uma rede credenciada gerou uma perda de benefício sem modificação do paradigma de tratamento e que a implementação de um serviço próprio gerou uma redução de custo e uma modificação no perfil assistencial (neste caso, mais voltado à promoção de saúde bucal e menos voltado a tratamentos cirúrgico-restauradores). Como existe uma grande preocupação com o crescimento dos gastos com saúde nas empresas 1,2,28, os resultados desse estudo nos levam a sugerir que a implantação de serviços próprios de odontologia baseado em evidências com profissionais e gerentes treinados para agir a partir dessa abordagem e com inclusão de tratamentos preventivos nas grandes empresas seja considerada e, conseqüentemente, mais estudada. 


\section{Resumo}

O presente estudo avaliou a assistência odontológica fornecida a cerca de 4 mil funcionários e dependentes de um hospital privado. A análise foi dividida em três momentos: (1) linha de base (controle): quando a assistência odontológica fornecida era terceirizada por uma empresa que operava com rede credenciada, (2) quando houve uma renegociação de preços com a prestadora original e (3) quando a assistência era feita por um serviço de odontologia próprio sem a intermediação de uma prestadora e com profissionais remunerados através de valores fixos. Foram coletados mensalmente dados econômicos e sobre o tipo e número de procedimentos realizados. A renegociação de preços reduziu os custos em cerca de $37 \%$ em relação à linha de base, ao passo que o serviço próprio reduziu os custos em 50\%. A renegociação de preços provocou uma diminuição de $31 \%$ no número de procedimentos realizados sem modificar o perfil da assistência, ao passo que o serviço próprio não causou diminuição na quantidade de serviços, mas modificou o padrão da assistência, pois se aumentaram os procedimentos relacionados com as causas das patologias e reduziramse os procedimentos cirúrgico-restauradores.

Planejamento em Saúde; Redução de Custos; Seguro Odontológico; Saúde Bucal

\section{Colaboradores}

L. C. Costa Filho coordenou o estudo, fez as análises econômicas e escreveu o artigo. B. B. Duncan e a C. A. Polanczyk participaram da orientação do estudo e revisaram o manuscrito. C. C. Costa e M. L. Sória participaram da implantação e execução do estudo. A. P. Habekost participou da coleta de dados dessa pesquisa.

\section{Agradecimentos}

Os autores gostariam de agradecer à Coordenação de Aperfeiçoamento de Pessoal de Nível Superior (CAPES) pelo apoio financeiro durante o doutorado do Dr. Luiz Cesar da Costa Filho e aos professores Ronaldo Bordin e Jandyra Fachel por suas sugestões na elaboração desse artigo. Os autores também gostariam de agradecer ao Hospital Moinhos de Vento e à empresa Salutaris por apoiarem esta pesquisa e permitirem o acesso a suas bases de dados.

\section{Referências}

1. Kaiser Family Foundation. Employer health benefits: 2005 annual survey. http://www.kff.org/ insurance/7315/ (acessado em 07/Mar/2007).

2. Rosenburg C. O insustentável custo da saúde nas empresas. Exame 2005; 3 ago.

3. Mercer Human Resource Consulting. Pesquisa Mercer Saúde 2003 - saúde corporativa. São Paulo: Mercer Human Resource Consulting; 2003.

4. Hewitt Associates LLC. Pesquisa Hewitt de Benefícios e Administração. São Paulo: Hewitt Associates LLC; 2004.

5. Hewitt Associates LLC. Pesquisa Hewitt de Benefícios e Administração. São Paulo: Hewitt Associates LLC; 2005.

6. Mercer Human Resource Consulting. Pesquisa Mercer Saúde 2004. São Paulo: Mercer Human Resource Consulting; 2004.
7. Agência Câmara de Notícias. Seguridade aprova assistência odontológica para empregado. http:// www2.camara.gov.br/internet/homeagencia/ma terias.html?pk=66255\&pesq=3520 $/ 04$ (acessado em 07/Mar/2007).

8. Soria ML. Remuneração de serviços de saúde bucal: formas e impactos na assistência. In: Schreiner M, Soria ML, Gottardo MCR, Franco JCQ, organizadores. Gestão financeira em saúde: remuneração e custos. Porto Alegre: Dacasa Editora; 2001. p. 39-91.

9. Grytten J. Models for financing dental services: a review. Community Dent Health 2005; 22:75-85.

10. Soria ML, Bordin R, Costa Filho LC. Remuneração dos serviços de saúde bucal: formas e impactos na assistência. Cad Saúde Pública 2002; 18:1551-9.

11 Guay AH. The implementation of managed care in dentistry. J Am Coll Dent 1995; 62:11-3. 
12. Brasil. Lei no. 9.656/98 de 3 de junho de 1998, dispõe sobre planos e seguros privados de assistência à saúde. Diário Oficial da União 1998; 4 jun.

13. Moysés SJ. O conceito de promoção de saúde na construção de sistemas de atenção em saúde bucal coletiva. In: Kriger L, organizador. Promoção de saúde bucal. 2a Ed. São Paulo: Editora Artes Médicas; 1999. p. 371-407.

14. Richards D, Lawrence A. Evidence based dentistry. Br Dent J 1995; 179:270-3.

15. Ministério da Saúde. Avaliação econômica em saúde: desafios para o Sistema Único de Saúde. http://portal.saude.gov.br/portal/arquivos/pdf/ livro_aval_econom_saude. pdf (acessado em 17/ Mar/2008)

16. Petitti DB. Advanced cost-effectiveness analysis. In: Petitti DB, editor. Meta-analysis, decision analysis and cost-effectiveness analysis: methods for quantitative synthesis in medicine. 2nd Ed. New York: Oxford University Press; 2000. p. 182-201.

17. Duncan BB, Schmidt MI. Medicina baseada em evidências. In: Duncan BB, Schmidt MI, Giugliani ERJ, organizadores. Medicina ambulatorial: condutas de atenção primária baseada em evidências. 3a Ed. Porto Alegre: Artmed Editora; 2004. p. 31-40.

18. Dumitresco A, Granados A, Wallace J, Watson S. Demand-driven evidence network in Europe. Bull World Health Organ 2006; 84:2.

19. Pinto VG. Saúde bucal coletiva. 4a Ed. São Paulo: Editora Santos; 2000.

20. Beazoglou TJ, Guay AH, Heffley DR. Capitation and fee-for-service dental benefit plans: economic incentives, utilization, and service-mix. J Am Dent Assoc 1988; 116:483-7.
21. Holloway PJ, Lennon MA, Mellor AC, Coventry P, Worthington HV. The capitation study. 1. Does capitation encourage "supervised neglect"? Br Dent J 1990; 168:119-21.

22. Lennon MA, Worthington HV, Coventry P, Mellor AC, Holloway PJ. The capitation study. 2. Does capitation encourage more prevention? Br Dent J 1990; 168:213-5.

23. Mellor AC, Coventry P, Worthington HV, Holloway PJ, Lennon MA. The capitation study. 3. The views of participating dentists and the profession. $\mathrm{Br}$ Dent J 1990;168:303-5.

24. Kriger L. Promoção de saúde bucal. 2a Ed. São Paulo: Editora Artes Médicas; 1999.

25. Mjor IA, Gordan VV. A review of atraumatic restorative treatment (ART). Int Dent J 1999; 49:127-31.

26. Scottish Intercollegiate Guidelines Network. Management of unerupted and impacted third molar teeth: report \# 43. http://www.sign.ac.uk/guide lines/fulltext/43/index.html (acessado em 07/ Mar/2007).

27. Coulter ID. Evidence-based dentistry and health services research: is one possible without the other? J Dent Educ 2001; 65:714-24.

28. Gabel J, Claxton G, Gil I, Pickreign J, Whitmore H, Finder B, et al. Health benefits in 2005: premium increases slow down, coverage continues to erode. The average cost of family coverage now exceeds the average yearly income of minimumwage Americans. Health Aff (Millwood) 2005; 24 1273-80.

Recebido em 04/Ago/2006

Versão final reapresentada em 21/Mar/2007

Aprovado em 25/Set/2007 